\title{
Matching procedure to morphology improves outcomes in neonates with tricuspid atresia
}

\author{
Tara Karamlou, MD, ${ }^{a, *}$ David A. Ashburn, MD, ${ }^{b, *}$ Christopher A. Caldarone, MD, ${ }^{a}$ Eugene H. Blackstone, MD, ${ }^{c}$ \\ Richard A. Jonas, MD, ${ }^{d}$ Marshall L. Jacobs, MD, ${ }^{e}$ William G. Williams, MD, ${ }^{a}$ Ross M. Ungerleider, MD, ${ }^{f}$ \\ and Brian W. McCrindle, MD, ${ }^{a}$ for the Members of the Congenital Heart Surgeons' Society
}

\begin{abstract}
From the Division of Cardiology and Cardiovascular Surgery, The Hospital for Sick Children, Toronto, Ontario, Canada, ${ }^{a}$ the Department of General Surgery, Wake Forest University School of Medicine, Winston-Salem, NC, ${ }^{\mathrm{b}}$ the Division of Cardiothoracic Surgery and Biostatistics and Epidemiology, Cleveland Clinic Foundation, Cleveland, Ohio, ${ }^{\mathrm{c}}$ the Division of Cardiovascular Surgery, Children's Hospital National Medical Center, Washington, DC, ${ }^{\text {d }}$ the Division of Cardiothoracic Surgery, St Christopher's Hospital for Children, Philadelphia, $\mathrm{Pa},{ }^{\mathrm{e}}$ and the Department of Cardiothoracic Surgery, Doernbecher Children's Hospital, Oregon Health and Science University, Portland, Ore. ${ }^{\mathrm{f}}$
\end{abstract}

Funding of the CHSS Data Center from member surgeons and institutions and the Hospital for Sick Children (Toronto) is acknowledged.

Read at the Eighty-fifth Annual Meeting of The American Association for Thoracic Surgery, San Francisco, Calif, April 10-13, 2005.

Received for publication April 4, 2005; revisions received June 21, 2005; accepted for publication July 23, 2005.

Address for reprints: Brian W. McCrindle, MD, The Hospital for Sick Children, 555 University Ave, Toronto, Ontario, Canada M5G 1X8 (E-mail: brian.mccrindle@ sickkids.ca)

*John W. Kirklin Fellow of the Congenital Heart Surgeons' Society Data Center, Toronto, Ontario, Canada.

J Thorac Cardiovasc Surg 2005;130:1503-10 $0022-5223 / \$ 30.00$

Copyright (C) 2005 by The American Association for Thoracic Surgery

doi:10.1016/j.jtcvs.2005.07.024
Objective: This study was undertaken to characterize morphologic substrate of tricuspid atresia with ventriculoarterial concordance and discriminate management strategies that lead to successful definitive repair.

Methods: From 1999 to 2004, a total of 150 babies with type I tricuspid atresia were enrolled from first diagnosis at 26 institutions. Antegrade pulmonary blood flow was absent in 19\%, restricted in 54\%, and unrestricted in 28\%. Competing-risk methodology determined the time-related prevalence and risk factors for death versus cavopulmonary anastomosis and subsequent death versus Fontan completion.

Results: Overall 5-year survival was $86 \%$. Initial palliation included systemicpulmonary arterial shunt in 64\%, pulmonary artery banding in $11 \%$, and cavopulmonary anastomosis in 24\%. Median age at cavopulmonary anastomosis was 6 months, with $83 \%$ undergoing bidirectional Glenn shunt and $17 \%$ undergoing hemi-Fontan procedure. By the age of 2 years, $89 \%$ had cavopulmonary anastomosis, $6 \%$ were dead, and $4 \%$ remained alive without cavopulmonary anastomosis. Risk factors for death without cavopulmonary anastomosis included presence of mitral regurgitation $(P=.03)$ and palliation with systemic-pulmonary arterial shunts not originating from the innominate artery $(P=.04)$. Factors associated with decreased transition rate to cavopulmonary connection included patient variables (younger admission age to a participating institution, noncardiac anomalies) and procedural variables (larger systemic-pulmonary arterial shunt diameter, previous palliation). Of patients undergoing cavopulmonary anastomosis, $75 \%$ had undergone a Fontan operation within 3 years.

Conclusion: Smaller shunt size and decreased pulmonary blood flow decrease mortality after initial palliation and increase the rate of successful transition to cavopulmonary anastomosis. Outcomes can be improved by placing smaller shunts from the innominate artery, especially in patients with any mitral regurgitation.

$\mathrm{T}$ ricuspid atresia with normally related great arteries (type I) is present in $70 \%$ of all patients with tricuspid atresia and is the most favorable form of univentricular heart necessitating the Fontan-Kreutzer operation. ${ }^{1,2}$ Patients with type I tricuspid atresia have an ideal subset of univentricular heart for which to define outcomes and associated risk factors because they are prevalent, have favorable outcomes, and are treated with a fairly uniform algorithm. The Congenital Heart Surgeons' Society (CHSS) therefore initiated a prospective study in neonates with type I tricuspid atresia to characterize the relation between morphology and management strategies leading to definitive repair.

\section{Patients and Methods}

Between January 1999 and November 2004, a total of 150 babies younger than 3 months with the diagnosis of type I tricuspid atresia were prospectively enrolled from 26 participating institutions. The number of patients enrolled per institution ranged from 1 to 12 , with four institutions enrolling 


\author{
Abbreviations and Acronyms \\ BDCPA = bidirectional cavopulmonary anastomosis \\ CHSS = Congenital Heart Surgeons' Society \\ LOS = length of stay \\ PA $=$ pulmonary artery \\ VSD $=$ ventricular septal defect
}

9 or more patients. Participation by member institutions was voluntary and confidential, and ethical approval was obtained per local requirements. The CHSS Data Center obtains annual approval from the Research Ethics Board of the Hospital for Sick Children, Toronto.

\section{Classification}

Type I tricuspid atresia is a congenital cardiac malformation with ventriculoarterial concordance in which the right atrium, in the setting of ventricular D-loop, fails to open into a ventricle through an atrioventricular valve..$^{3-6}$ Type I tricuspid atresia is subdivided according to degree of obstruction to pulmonary blood flow: type Ia has no antegrade pulmonary blood flow because of pulmonary atresia $(\mathrm{n}=$ 28, 19\%), type Ib has restricted pulmonary blood flow because of either pulmonary artery (PA) trunk hypoplasia or restrictive ventricular septal defect (VSD, $\mathrm{n}=80,54 \%$ ), and type Ic has unrestricted pulmonary blood flow because of a nonrestrictive VSD and absence of pulmonary stenosis or atresia $(n=41,28 \%){ }^{6}$

\section{Data Collection}

Demographic and morphologic characteristics of all patients are given in Table E1 and Appendix E1.

Demographic data. Prospective data were collected from submitted medical records. The study is ongoing, and the most recent cross-sectional follow-up was performed between September and November 2004. Follow-up was complete for 132 patients (88\%).

Morphologic data. Echocardiographic and catheterizationderived cardiac structural variables, such as right and left PA dimensions, were converted into $Z$ scores. ${ }^{7,8}$ A composite mean PA $Z$ score was then created as follows: ( $Z$ score right PA $+Z$ score left PA)/2. Initial echocardiographic assessment was available for 140 patients (93\%). Initial valvular pathology included mitral regurgitation in 32 cases (34\%), aortic regurgitation in $13(10 \%)$, and aortic stenosis in 4 (3\%). Follow-up echocardiographic or catheterization assessment was available in 119 patients (79\%) before bidirectional cavopulmonary anastomosis (BDCPA). Valvular pathology developed in 24 patients, including mitral regurgitation in 19 , aortic regurgitation in 1 , and systemic outflow tract stenosis in 4 .

\section{Data Analysis}

Because surgical management of tricuspid atresia generally requires a sequence of procedures (Figure E1), identification of the relationships between the morphologic substrate and those components of the management strategy that ultimately lead to a successful Fontan procedure mandates an analytic approach that considers results of intermediate, as well as final, stages of repair. Thus in this analysis we did the following: (1) determined the proportion of patients reaching defined end states (BDCPA, Fontan completion, death), (2) identified risk factors modulating transition into each end state, and (3) created from these factors models capable of predicting the number of patients reaching each defined end state given specific values for these risk factors.

Multiphase parametric modeling of the hazard function and competing risks methodology were used to determine rates of transition to mutually exclusive time-related events and to identify incremental risk factors associated with each transition rate, as previously described. ${ }^{9,10}$ Linearizing transformations including the addition of quadratic terms for continuous predictors, and interactions among retained variables in the model were considered in all cases. Variable selection was primarily by bootstrap bagging. ${ }^{11}$

Characteristics of patients at palliation, BDCPA, and Fontan operation are given in Appendix E1 and Tables E2 through E4. In addition, multivariable linear regression was performed to determine the relationship between systemic-pulmonary arterial shunt characteristics, such as diameter and origin, and systemic oxygen saturation after shunt placement, controlling for other sources of pulmonary blood flow. This was accomplished by incorporating the status of the ductus arteriosus and the presence of antegrade flow through the main PA as binary regressor variables in the model concomitant with the shunt variables of origin and diameter.

Data are presented as frequency, median with range, or mean \pm $\mathrm{SD}$ as appropriate, with the numbers of missing values indicated. Percentages, hazard functions, and competing risk estimates are presented with confidence intervals equivalent to $1 \mathrm{SE}(68 \%)$. All data analyses were performed with SAS statistical software (version 9; SAS Institute, Inc, Cary, NC).

\section{Results}

\section{Overall Mortality}

There were 17 deaths: 9 occurred after systemic-pulmonary arterial shunt operations, 6 after BDCPA, and 2 after Fontan completion. Overall survival at 5 years was $86 \%(70 \%$ confidence interval 82\%-89\%; Figure E2).

\section{Prevalence, Timing, and Factors Predictive of End States}

By 2 years from diagnosis, $6 \%$ of patients had died without BDCPA, $89 \%$ had undergone BDCPA, 4\% remained alive without BDCPA or further operation, and $1 \%$ had undergone single-stage Fontan procedures (Figure 1).

Death without BDCPA $(\boldsymbol{n}=9)$. The time-related hazard function for death without BDCPA was characterized by an early phase responsible for all nine events. Incremental risk factors included the presence of mitral regurgitation on initial echocardiogram (coefficient $1.56 \pm 0.70 ; P=.03$ ) and systemic-PA shunt placement not originating from the innominate artery (coefficient $1.64 \pm 0.79 ; P=.04$; Table 1). Figure 2 shows that patients who had shunts originating from the innominate artery had substantially better survival than did those with shunts from the subclavian artery or other sites. The benefit of an innominate artery shunt was magnified in the presence of mitral regurgitation, as seen in Figure E3).

$\boldsymbol{B D C P A}(\boldsymbol{n}=\mathbf{1 2 8})$. The median age at BDCPA was 5.7 months (range 1.7 months-2 years). The time-related hazard for achievement of BDCPA was characterized by a single early 


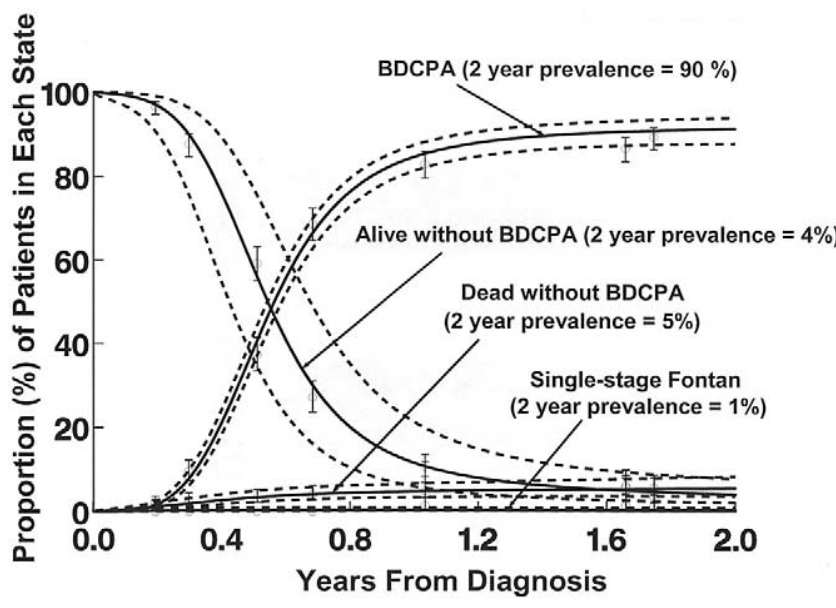

Figure 1. Competing risks depiction of events after diagnosis in 150 patients with tricuspid atresia. All patients began alive and thereafter migrated to one of four mutually exclusive end states (death, BDCPA, single-stage Fontan completion, or remaining alive without BDCPA) at time-dependent rates defined by underlying hazard functions. At any point in time, sum of proportions of children in each state is $100 \%$. For example, estimated prevalences after 2 years from diagnosis are as follows: $89 \%$ BDCPA; $6 \%$ dead without BDCPA, $4 \%$ alive without BDCPA, and $1 \%$ single-stage Fontan completion. Solid lines represent parametric point estimates; dashed lines enclose $\mathbf{7 0} \%$ confidence intervals; circles with error bars represent nonparametric estimates; numbers in parentheses indicate estimated proportions of patients in each state at 2 years from diagnosis.

phase responsible for all 128 events. Three patient-specific factors (older age at admission, absence of either smaller or larger composite PA $Z$ score on admission echocardiogram, and absence of noncardiac anomalies) and two procedural variables (smaller absolute systemic-pulmonary arterial shunt

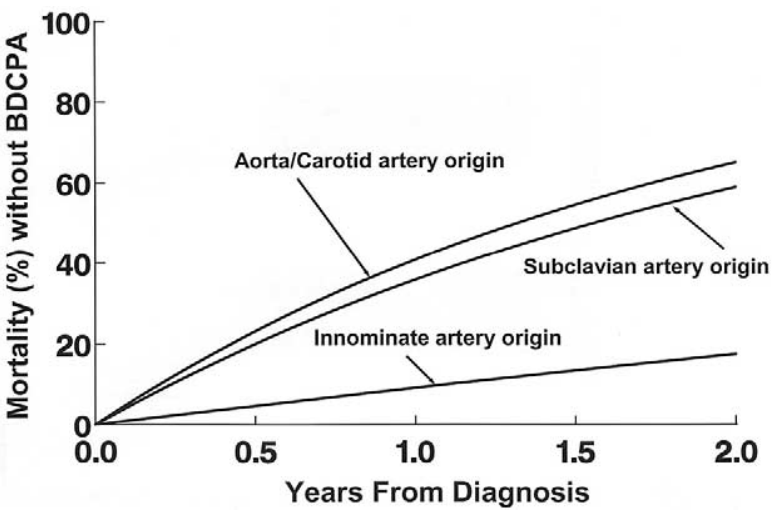

Figure 2. Mortality without BDCPA stratified by shunt origin. Patients who had systemic-pulmonary arterial shunts originating from innominate artery had significantly improved survival relative to those with shunts from subclavian artery or other sites, which carried nearly equivalent mortality rates.

diameter [Figure E4] and absence of palliation before BDCPA) were associated with a higher likelihood of surviving from initial diagnosis to BDCPA (Table 1). The risk-adjusted competing risk diagrams shown in Figure 3 illustrate that when a systemic shunt is required, placing a smaller shunt decreases mortality and increases the rate of transition to BDCPA.

The second competing risks analysis for events after BDCPA predicted that at 3 years from BDCPA, 5\% would have died without a Fontan operation, $75 \%$ would have subsequent Fontan operation, and 20\% would remain alive without Fontan or other operation (Figure 4).

Death after BDCPA without Fontan completion ( $n=$ 6). The time-related hazard function for death after BDCPA without conversion to Fontan circulation was characterized by a steep early phase accounting for 3 events and an ongoing late phase accounting for 3 events.

TABLE 1. Incremental risk factors for time-related transition to end states after diagnosis $(n=150)$

\begin{tabular}{|c|c|c|c|}
\hline Variable & Coefficient ( \pm SE) & $P$ value & Reliability* (\%) \\
\hline \multicolumn{4}{|l|}{ For death without BDCPA } \\
\hline Mitral valve regurgitation at initial echocardiogram & $1.56 \pm 0.70$ & .03 & $60 \%$ \\
\hline Non-innominate artery systemic-pulmonary arterial shunt origin & $1.64 \pm 0.79$ & .04 & $64 \%$ \\
\hline \multicolumn{4}{|l|}{ For survival to BDCPA } \\
\hline Smaller absolute systemic-pulmonary arterial shunt size (per $1 \mathrm{~mm}) \dagger$ & $7.17 \pm 4.74$ & .13 & $72 \%$ \\
\hline Older age at admission (per 1 y) & $0.62 \pm 0.37$ & .08 & $62 \%$ \\
\hline Absence of larger or smaller PA Z score at initial echocardiogram & $\ddagger$ & .08 & $59 \%$ \\
\hline No previous palliation & $0.66 \pm 0.31$ & .03 & $62 \%$ \\
\hline No systemic-pulmonary arterial shunt & $0.42 \pm 0.28$ & .10 & $72 \%$ \\
\hline Absence of noncardiac anomalies & $0.62 \pm 0.25$ & .02 & $60 \%$ \\
\hline \multicolumn{4}{|l|}{ For survival to Fontan completion } \\
\hline Restrictive VSD on cavopulmonary connection echocardiogram & $0.81 \pm 0.48$ & .09 & $62 \%$ \\
\hline
\end{tabular}

*Reliability determined by bootstrap bagging (variable resampling) method. $†$ After inverse transformation. $\ddagger$ Complex polynomial function including two terms, mean combined PA $Z$ score and square of square transformation of mean PA $Z$ score. 


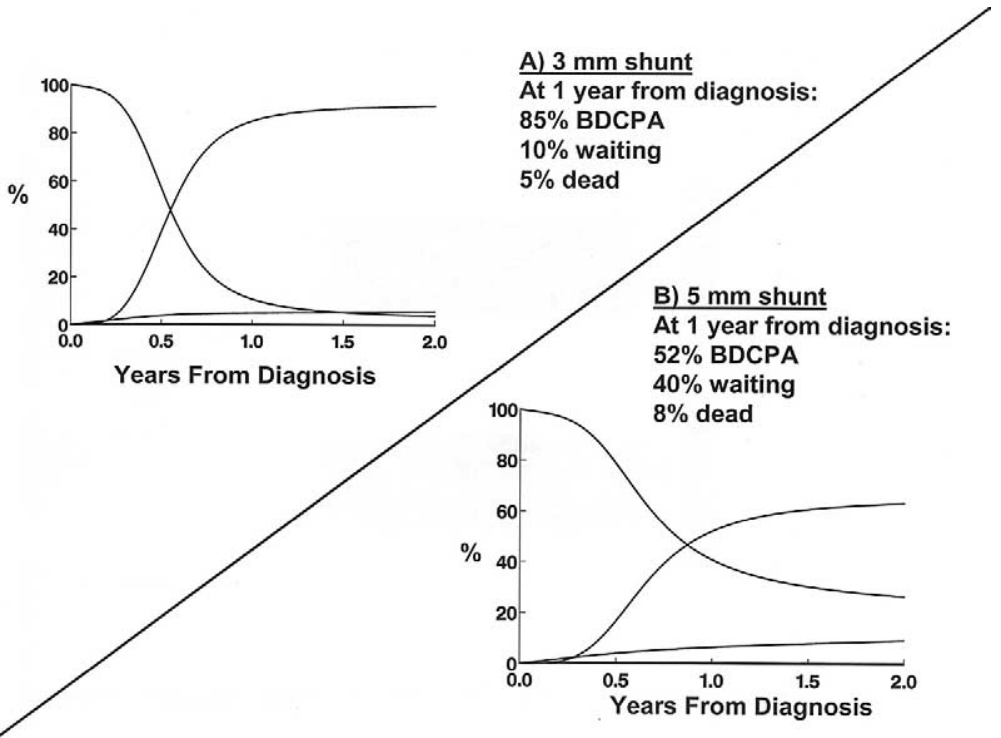

Figure 3. Smaller systemic-pulmonary arterial shunt size resulted in decreased mortality and increased transition rates to BDCPA. A, Use of 3-mm shunt resulted in $85 \%$ of patients having BDCPA by 1 year, $10 \%$ still in palliated state, and only $5 \%$ of dead. $B$, Use of larger 5-mm shunt resulted in much slower transition rate and slightly increased rate of death without BDCPA.

Fontan completion $(\boldsymbol{n}=74)$. The median age at Fontan completion was 2.5 years (range 1-5 years). The time-related hazard function for conversion to Fontan circulation was characterized by a single early phase accounting for all 74 events. The presence of a restrictive VSD on pre-BDCPA echocardiography was associated with a greater proportion of neonates reaching Fontan completion (Table 1). The small event num-

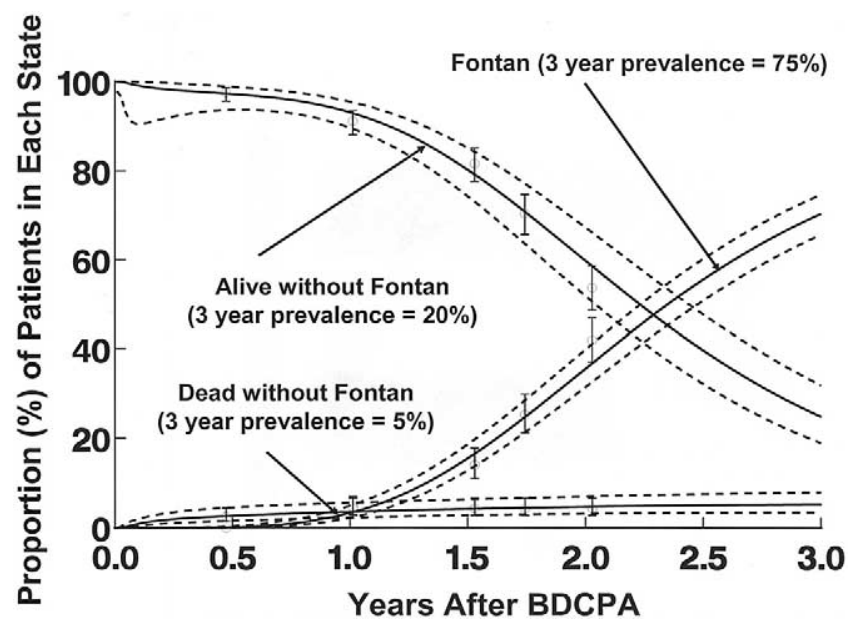

Figure 4. Competing-risks depiction of events after BDCPA in 128 patients who underwent BDCPA. Estimated prevalences after 3 years from BDCPA are as follows: $\mathbf{7 5} \%$ Fontan completion, $20 \%$ alive without Fontan conversion, and $5 \%$ dead without Fontan completion. Solid lines represent parametric point estimates; dashed lines enclose $70 \%$ confidence intervals; circles with error bars represent nonparametric estimates; numbers in parentheses indicate estimated proportions of patients in each state at 3 years from BDCPA. ber precluded the identification of associated risk factors for death before Fontan completion.

\section{Management of Competing Sources of Pulmonary Blood Flow}

Main PA trunk ligation occurred in 11 patients at the time of systemic shunt placement: 8 of these were type Ic and 3 were type Ib. There were 15 deaths among 121 patients with flow through the main PA (types Ib and Ic) and 14 deaths among the 68 patients in this group who had a systemic-pulmonary arterial shunt. Eleven of these $(16 \%)$ occurred in those without main PA trunk ligation, and $3(4 \%)$ occurred in those with ligation $(P=.21)$. Thus, there was a trend toward improved survival among those patients undergoing main PA trunk ligation at the time of systemic shunt placement, although it did not reach statistical significance. It is possible that this benefit is increased in patients with any degree of mitral valve regurgitation, provided the valve is anatomically normal and the regurgitation is related to ventricular volume loading. New mitral regurgitation was present in 19 patients at the time of BDCPA, 14 of whom had preservation of native PA flow and 5 of whom had main PA trunk ligation. There was a trend toward an increased prevalence of mitral regurgitation among those without ligation (74\% vs $26 \%, P=.18$ ).

\section{Influence of Age at BDCPA}

The ideal age for transition to BDCPA could not be precisely determined by our analysis, because patient age at BDCPA was not associated with either death after BDCPA or survival to Fontan completion. Because the risk factors identified in our analysis were associated with earlier rates of transition, however, we explored whether early BDCPA was correlated with other adverse end points, including 


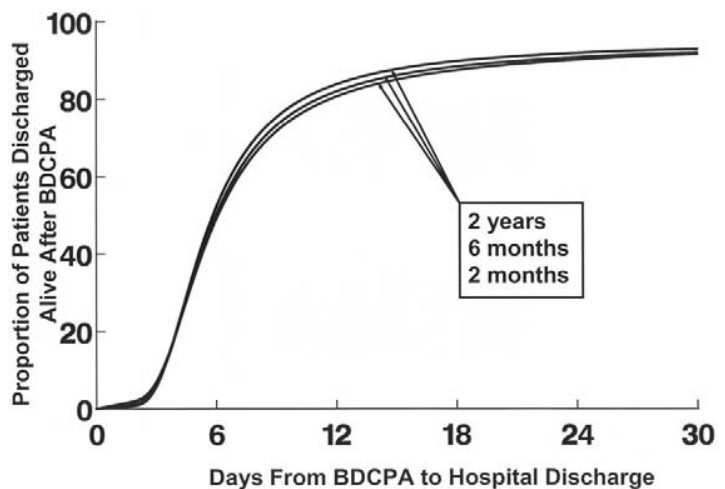

Figure 5. Proportion of patients discharged alive after BDCPA stratified by patient age at BDCPA. Of 128 patients undergoing BDCPA, 126 were discharged alive. Multivariable competing risks equation for survival to hospital discharge was solved for hypothetic patient weighing $3.7 \mathrm{~kg}$ with previous palliation at three different values for age at BDCPA. Age at BDCPA did not influence either hospital LOS or in-hospital mortality. Youngest patients in our study undergoing BDCPA (at about 2 months) fared no worse than did those of median or older age.

death before hospital discharge or increased hospital length of stay (LOS) after BDCPA. The median LOS was 6 days (range 3-351 days). There were 2 in-hospital deaths, 1 occurring 2 weeks after BDCPA and the other occurring after a prolonged LOS of nearly 1 year. A separate competing risks analysis showed that 60 days after BDCPA, $95 \%$ had been discharged alive, $4 \%$ were alive but remained in the hospital, and 1\% had died without discharge (Figure E5). Age at BDCPA was not significantly associated with either competing outcome, and therefore younger or older age at BDCPA did not increase either LOS or in-hospital mortality (Figure 5).

\section{Discussion}

\section{Mortality}

Previous reports have shown that outcomes in infants with tricuspid atresia and normally related great arteries are considerably better than for other morphologic substrates of singleventricle physiology. ${ }^{12-14}$ We have shown that mortality occurs primarily at the initial palliation stage before BDCPA and that outcomes beyond transition to BDCPA are excellent.

The presence of mitral regurgitation at initial echocardiography (34\%) and systemic-pulmonary arterial shunts not originating from the innominate artery were associated with death without conversion to BDCPA. The association between systemic atrioventricular valve regurgitation and adverse outcome in patients with single-ventricle physiology is well known. ${ }^{15-17}$ The deleterious effects of significant mitral regurgitation may be potentiated by pulmonary overcirculation related to the maintenance of additional sources of competing pulmonary blood flow. Ventricular unloading has been shown to be reduced in this setting. ${ }^{18}$ Patients in our study who had undergone palliation and had antegrade pulmonary blood flow tended to have an increased prevalence of mitral regurgitation (74\%) before BDCPA relative to those who underwent main PA trunk ligation (26\%). Although we cannot provide conclusive evidence that PA trunk ligation is the optimal strategy in all cases, our data show that reduced pulmonary blood flow may lead to improved outcomes, especially in patients with any degree of mitral regurgitation.

The influence of shunt origin on mortality risk is complex, because the shunt may be only one determinant of total pulmonary blood flow. However, innominate artery shunts had a smaller indexed size $(1.15 \mathrm{~mm} / \mathrm{kg})$ than did those originating from alternate sites, reflecting preferential use of $3.5-\mathrm{mm}$ shunts (as opposed to 4.0-mm shunts) in this population. Smaller shunt size is consistent with previous data from a canine model of univentricular heart that showed systemicpulmonary arterial shunts with indexed size 0.8 to 1.0 to result in more favorable outcomes. ${ }^{19}$ In agreement with these findings, our multivariable analysis demonstrated that patients with innominate artery shunts had lower postoperative oxygen saturation after controlling for other sources of pulmonary blood flow, indicating a reduction in the shunt contribution to total pulmonary blood flow. Appropriate shunt sizing therefore may mitigate the tendency toward excessive shunt flow, because the predominant determinant of flow becomes the diameter of the smaller shunt, rather than the diameter of the larger originating vessel. This finding was confirmed recently in a computational fluid dynamic model in which innominate artery diameter and insertion angle had negligible influence on pressure-flow relationships. ${ }^{20}$

A previous report from Sittiwangkul and colleagues ${ }^{13}$ has suggested that central shunt origin in patients with tricuspid atresia may predispose toward pulmonary overcirculation and contribute to PA distortion. The disadvantage imparted by non-innominate artery origin, however, may be a surrogate for other morphologic or technical covariates, in that the selection of alternate shunt origination site in our series often reflected the presence of other unfavorable anatomic characteristics that precluded the use of the innominate artery.

\section{Transition to BDCPA}

The favorable influence of older presenting age on outcomes in patients with tricuspid atresia has been previously described $^{12,13,21}$ and may be related to natural selection. Patients in our study who were admitted at an older age generally had balanced physiology and therefore underwent BDCPA without previous palliation.

Our data showing that smaller systemic-pulmonary arterial shunt diameter favors transition to BDCPA is concordant with a previous CHSS report on patients with hypoplastic left heart syndrome in which lower post-Norwood oxygen saturation 
and smaller indexed shunt size were also associated with increased rates of transition. ${ }^{14}$ Thus smaller shunt size and less total pulmonary blood flow not only decrease mortality after initial palliation but also increase the rate of successful transition to BDCPA.

The optimal timing for BDCPA has not been conclusively determined, in part because general consensus dictates that transition occur between 3 months and 1 year, so there are few patients undergoing BDCPA at extremes of age. In addition, the scarcity of adverse events (death or not reaching Fontan completion) among patients with type I tricuspid atresia hampers risk-factor identification. In our study, the median age at BDCPA was 6 months, and the procedure was almost universally performed after the age of 2 months (with 2 exceptions, at 1.7 and 1.8 months) and rarely after 1 year. Patient age at BDCPA was not a predictor of end state achievement, nor was it associated with increased hospital LOS. However, an important corollary is that no significant disadvantage was imparted by earlier BDCPA transition. Valid arguments exist in support of earlier conversion, including optimization of pulmonary/ systemic blood flow ratio, decreased single-ventricle volume load, and preservation of PA architecture. ${ }^{22}$ The long-term myocardial benefits of early volume load reduction have important implications, because myocardial failure is among the most important causes of morbidity and mortality among patients with univentricular heart independent of Fontan completion. ${ }^{23}$ Two clinical studies have shown acceptable outcomes in patients undergoing BDCPA between 2 and 6 months but significantly increased mortality and BDCPA takedown rate when the procedure was attempted before the age of 2 months. ${ }^{22,24}$ The benefits of limited pulmonary blood flow and early conversion must therefore be weighed against the risk of premature BDCPA performance, which according to our and others' data may potentially be defined as 2 months of age. ${ }^{24}$

\section{Study Limitations}

Although this study represents the largest prospective cohort of patients with tricuspid atresia, and a comprehensive set of variables was used, lack of statistical power and missing data points are potential weaknesses. The current data set did not include sufficient interstage longitudinal measures of PA and ventricular size, nor were there sufficient quantitative valvular or ventricular function indices. Incorporation of this data could have allowed determination of the influence of different management strategies, particularly related to accessory pulmonary blood flow, on growth of anatomic structures and singleventricle performance.

\section{Conclusions}

We have shown that demographic, morphologic, and procedural characteristics have important influences on the proportion of patients reaching each end state and that outcomes beyond conversion to BDCPA are excellent. Results in babies with tricuspid atresia may be improved by placing systemic shunts from the innominate artery, using the smallest systemic shunt possible to achieve adequate oxygen saturation, and interrupting competing sources of pulmonary blood flow in patients with any degree of mitral regurgitation. Delaying BDCPA beyond 2 months of age confers no discernible benefit with regard to hospital LOS, mortality, or achievement of Fontan completion.

We thank all of the members of the CHSS and their pediatric cardiology colleagues for their contributions to this study. We acknowledge the assistance of personnel from the CHSS Data Center, particularly Geraldine Cullen-Dean, Sally Cai, Olga Levesque, and Candice Cumberbach for coordinating the collection and management of these data.

\section{References}

1. Fontan F, Baudet E. Surgical repair of tricuspid atresia. Thorax. 1971;26:240-8.

2. Kreutzer G, Galindez E, Bono H, DePalma C, Laura JP. An operation for the correction of tricuspid atresia. J Thorac Cardiovasc Surg. 1973;66:613-21.

3. Jacobs ML, Mayer JE Jr. Congenital Heart Surgery Nomenclature and Database Project: single ventricle. Ann Thorac Surg. 2000;64(4 Suppl):S197-204.

4. Edwards JE, Burchell HB. Congenital tricuspid atresia: a classification. Med Clin North Am. 1949;33:1177-8.

5. Tandon R, Edwards JE. Tricuspid atresia. A re-evaluation and classification. J Thorac Cardiovasc Surg. 1974;67:530-42.

6. Kouchoukos NT, Blackstone EH, Doty DB, Hanley FL, Karp RB. Tricuspid atresia and ,management of single-ventricle physiology. In: Kouchoukos NT, Blackstone EH, Doty DB, Hanley FL, Karp RB, editors. Kirklin/Barratt-Boyes cardiac surgery. Morphology, diagnostic criteria, natural history, techniques, results, and indications. 3rd ed. Philadelphia: Elsevier Science; 2003. p 1113-75.

7. Daubeney PE, Blackstone EH, Weintraub RG, Slavik Z, Scanlon J, Webber SA. Relationship of the dimension of cardiac structures to body size: an echocardiographic study in normal infants and children. Cardiol Young. 1999;9:402-10.

8. Sluysmans T, Colan SD. Theoretical and empirical derivation of cardiovascular allometric relationships in children. J Appl Physiol. 2005;99:445-57.

9. Blackstone EH, Naftel DC, Turner ME Jr. The decomposition of time-varying hazard into phases, each incorporating a separate stream of concomitant information. J Am Stat Assoc. 1986;81:615-24.

10. McGriffin DC, Naftel DC, Kirklin JK, Morrow WR, Towbin J, Shaddy $\mathrm{R}$, et al. Predicting outcome after listing for heart transplantation in children: comparison of Kaplan-Meier and parametric competing risk analysis. Pediatric Heart Transplant Study Group. J Heart Lung Transplant. 1997;16:713-22.

11. Blackstone EH, Rice TW. Clinical-pathologic conference: Clinicalpathologic conference: use and choice of statistical methods for the clinical study, "superficial adenocarcinoma of the esophagus." J Thorac Cardiovasc Surg. 2001;122:1063-76.

12. Franklin RC, Spiegelhalter DJ, Sullivan ID, Anderson RH, Thoele DG, Shinebourne EA, et al. Tricuspid atresia presenting in infancy. Survival and suitability for the Fontan operation. Circulation. 1993; 87:427-39.

13. Sittiwangkul R, Azakie A, Van Arsdell GS, Williams WG, McCrindle BW. Outcomes of tricuspid atresia in the Fontan era. Ann Thorac Surg. 2004;77:889-94.

14. Ashburn DA, McCrindle BW, Tchervenkov CI, Jacobs ML, Lofland GK, Bove EL, et al. Outcomes after the Norwood operation in neonates with critical aortic stenosis or aortic valve atresia. $J$ Thorac Cardiovasc Surg. 2003;125:1070-82.

15. McCrindle BW, Tchervenkov CI, Konstantinov IE, Williams, WG, Neirotti RA, Jacobs ML, et al. Risk factors associated with mortality 
and interventions in 472 neonates with interrupted aortic arch: a Congenital Heart Surgeons' Society study. J Thorac Cardiovasc Surg. 2005;129:343-50.

16. Ashburn DA, Blackstone EH, Wells WJ, Jonas RA, Pigula FA, Manning $\mathrm{PB}$, et al. Determinants of mortality and type of repair in neonates with pulmonary atresia and intact ventricular septum. $J$ Thorac Cardiovasc Surg. 2004;127:1000-8.

17. Gentles TL, Mayer JE, Gavreau K, Newburger JW, Lock JE, Kupferschmid JP, et al. Fontan operation in five hundred consecutive patients: factors influencing early and late outcome. J Thorac Cardiovasc Surg. 1997; 114:1-20.

18. Uemura H, Yagihara T, Kawashima Y, Okada K, Kamiya T, Anderson $\mathrm{RH}$. Use of the bidirectional Glenn procedure in the presence of forward flow from the ventricles to the pulmonary arteries. Circulation. 1995;92(9 Suppl):II228-32.

19. Kitaichi T, Chikugo F, Kawahito T, Hori T, Masuda Y, Kitagawa T. Suitable shunt size for regulation of pulmonary blood flow in a canine model of univentricular parallel circulations. J Thorac Cardiovasc Surg. 2003;125:71-8.

20. Migliavacca F, Pennati G, Dubini G, Fumero R, Pietrabissa R, Urcelay G, et al. Modeling of the Norwood circulation: effects of shunt size, vascular resistances, and heart rate. Am J Physiol Heart Circ Physiol. 2001;280:H2076-86.

21. Cleveland DC, Kirklin JK, Naftel DC, Kirklin JW, Blackstone EH, Pacifico AD, et al. Surgical treatment of tricuspid atresia. Ann Thorac Surg. 1984;38:447-57.

22. Chang AC, Hanley FL, Wernovsky G, Rosenfeld HM, Wessel DL, Jonas RA, et al. Early bidirectional cavopulmonary shunt in young infants. Postoperative course and early results. Circulation. 1993; 88(5 Pt 2):149-158

23. Schwartz SM, Gordon D, Mosca RS, Bove EL, Heidelberger KP, Kulik TJ. Collagen content in normal, pressure, and volume overloaded developing human hearts. Am J Cardiol. 1996;77:734-8.

24. Reddy VM, McElhinney DB, Moore P, Haas GS, Hanley FL. Outcomes after bidirectional cavopulmonary shunt in infants less than 6 months old. J Am Coll Cardiol. 1997;29:1365-70.

\section{Discussion}

Dr Hillel Laks (Los Angeles, Calif). Dr Karamlou, that was a beautifully presented article. I also want to give my thanks and credit to the Congenital Heart Surgeons' Society, to Dr Williams, who runs the data center, and to the rest of the group that reviewed and presented these data.

At UCLA at and many other centers, the ideal management of tricuspid atresia, or single-ventricle heart, would include the following: control of pulmonary blood flow at an early age, preferably younger than 1 month, which would include reducing flow if it is excessive or providing a shunt if it is not; a cavopulmonary connection at approximately 3 months regardless of whether there are adequate oxygen saturations and an adequate shunt or blood flow; and a Fontan operation preferably at 4 to 5 years of age.

All the deaths before cavopulmonary connection occurred after the systemic-pulmonary arterial shunt. The data also shows that mitral regurgitation was associated with most of the mortality, and larger polytetrafluoroethylene shunt size was associated with a delayed cavopulmonary shunt.

Dr Karamlou, was there a cohort who received early control of pulmonary blood flow and a cavopulmonary connection electively at 3 months of age who might be compared with a group who did not, to look at the question as to whether a rigidly controlled method of treatment at each stage would result in a better outcome?

Dr Karamlou. Regarding your question about performing a controlled study, this was a prospective observational study, and we therefore made no attempt to mandate management at any of the participating institutions.

Regarding the management of pulmonary blood flow, most patients without pulmonary atresia who received systemic-pulmonary arterial shunts actually had maintenance of antegrade flow through the native PA. Cavopulmonary connection was almost universally performed between 2 months and 1 year of age. The variable of age at cavopulmonary connection was analyzed as a continuous variable, and we therefore did not dichotomize or categorize it, nor did we use a particular age to define whether 3 versus 4 months was the ideal age for performing cavopulmonary connection.

I can say, however, that the youngest patient in our study who underwent cavopulmonary connection was aged 1.7 months. There have been other articles which suggest that earlier cavopulmonary connection may be beneficial as long as it's not performed before 2 months of age, and I think our data tend to agree with this point.

Dr Laks. Another interesting finding was the apparent importance of the point of origin for a shunt, with better results with the innominate artery origin than with the subclavian artery, aorta, or carotid artery. There was also a correlation with delayed cavopulmonary shunt and shunt size, suggesting that patients were followed up as long as their oxygen saturations were adequate.

Were the larger shunts used in the procedures that were not from the innominate artery in origin, subclavian and aortic, mostly the large polytetrafluoroethylene grafts that were used, relative to the innominate group, and would this explain the difference? Because there was a time when for subclavian shunts it was suggested that the proximal subclavian would control flow and it was therefore okay to use a 5-mm polytetrafluoroethylene graft, which we in our own experience have found not to be acceptable and to result in overcirculation. So was this specific to institutional choice of shunt size rather than point of origin? And was it related to polytetrafluoroethylene shunt size rather than point of origin?

Dr Karamlou. As you have alluded to, trying to dissect out the effect of shunt size from the overall contribution of pulmonary blood flow is complex, because the shunt is only one determinant of total pulmonary blood flow. Most of the shunts that were placed from the innominate artery were actually downsized to $3.5 \mathrm{~mm}$, compared with a 4-mm or larger shunt that was generally placed from an alternative site, either the subclavian artery or centrally from the aorta.

There have been data from a canine model of univentricular heart to suggest that an optimal indexed shunt size to body weight close to $1 \mathrm{~mm} / \mathrm{kg}$ provides the best matching between pulmonary and systemic blood flows. The innominate artery shunts were closer to this value at 1.1 than were the larger indexed shunt size used for either the subclavian or the aortic point of origin. So I do think that perhaps the innominate artery origin may be a surrogate for smaller shunt size, but it also may be a surrogate for more favorable morphologic type. For instance, in some cases, an aberrant right subclavian artery or exceedingly small head vessels precluded the use of the innominate artery. This could explain the favorable influence of the site of origin.

Dr Laks. I think it would be worth clarifying this, because the conclusion drawn that these other sites of origin are intrinsically not good might not be warranted by the data because of institutional bias.

At UCLA, most cavopulmonary connections are done off pump; only a small minority are done on pump, and only if other procedures 
are done or if the total pulmonary blood flow would be occluded by the application of clamps with cannulation of the superior vena cava and the right atrial appendage. In this study, $70 \%$ of connections were done with bypass, and $17 \%$ were done with circulatory arrest. It would be interesting to compare the morbidity, including neurologic effects and blood use, and the cost between these two groups. Certainly, medicolegal issues, even if not substantiated, could be raised with the use of circulatory arrest for a procedure that could be done without it.

Do you have data regarding any difference in morbidity and mortality between the on-pump and off-pump groups?

Dr Karamlou. We didn't specifically look at on-pump versus off-pump procedures, because the numbers of cases performed off pump was actually very small. The patients who had circulatory arrest were those who underwent a hemi-Fontan operation as opposed to a bidirectional Glenn shunt.

I do agree that looking at neurologic morbidity as well as cost might be useful in the future. As I noted in the beginning, this is an ongoing study, and enrollment continues. So perhaps in the next couple of years, if everybody keeps enrolling their patients, we may have sufficient data to answer this question.

Dr Laks. My final comment and question regard the controversy as to whether at the time of cavopulmonary connection an additional source of pulmonary blood flow should be left. At UCLA, we have made it our practice as often as possible to leave an additional source of pulmonary blood flow, and that would be a tightened band or a shunt made slightly smaller. The advantage, theoretically, is that you would have a total pulmonary/systemic blood flow ratio aimed at 1.0 to 1.3. And because the superior vena cava only provides a pulmonary to systemic blood flow ratio of 0.35 , you would have a normal amount of pulmonary blood flow, and not an excessive amount or too small an amount, and could delay the Fontan completion until about 4 or 5 years of age, when an adult-sized conduit could be used. In addition, during that time, the child would have a chance to respond to exercise, because systemic hypertension during exercise would increase pulmonary blood flow.

In this particular study, were you able to look at those who did have an additional source of flow and those who did not, and whether this influenced outcome and what influence it had on the time of Fontan completion?

Dr Karamlou. The issue of competing sources of pulmonary blood flow was a question we initially had tremendous interest in exploring, for the reasons that you mentioned. There is significant controversy in the literature regarding this issue. Certain authors have advocated that leaving an additional source of pulmonary blood flow reduces arteriovenous malformation formation and, as you say, may also postpone the need for Fontan operation to a later age. However, Mainwaring and colleagues have indicated that leaving additional sources of pulmonary blood flow in their series of 92 patients actually increased morbidity and had an unfavorable impact on outcomes.

In our study, patients who had an additional source of pulmonary blood flow, usually antegrade flow through the native PA, had an increased incidence of mitral regurgitation, which portended poorer outcomes in our current series. Two other papers, one from my own institution, showed that delaying the age at Fontan operation actually led to worse outcomes after Fontan operation. Although the study reported here did not focus on post-Fontan outcomes, this is something that we will certainly explore at as the cohort matures.

Dr Charles D. Fraser, Jr (Houston, Tex). Congratulations on an excellent analysis, but I wonder if you might explore another inference. If I understand your data correctly, the patients who died with shunts in place were older, meaning a longer transition time from the newborn period to the cavopulmonary anastomosis. Our friends from Wisconsin have taught us that in a more complicated singleventricle model, the hypoplast, a close surveillance program decreases interstage mortality. Isn't this just another example of interstage mortality, and shouldn't one of the inferences be that we need to follow all our single-ventricle patients much more closely and perhaps refer them for cavopulmonary anastomosis earlier?

Dr Karamlou. That is a good point. It also raises another interesting point: although tricuspid atresia with normally related great arteries has favorable morphologic type compared with other forms of univentricular heart, there is still significant interstage mortality. I agree that a surveillance program for all single-ventricle patients would probably lead to improved outcomes.

Dr Christo I. Tchervenkov (Montreal, Quebec, Canada). Congratulations, I enjoyed your presentation. I wonder if you can give us your thoughts on the fact that, unlike in this article or this cohort of patients with tricuspid atresia, where age at Glenn shunt was perhaps associated with better outcome, in a similar cohort of patients with hypoplastic left heart syndrome after the Norwood operation, the opposite was found. That, is younger age at Glenn shunt was associated with a higher mortality. How do you contrast these two different findings? Can you explain them? Could perhaps a comparison study be undertaken to try to tease out why in this series young age at Glenn was better and in the either series was not as good?

Dr Karamlou. I cannot speculate as to exactly what the differences are because there was no comparison made. I believe an important distinction between the two groups is that in the Norwood series, all of the patients were completely shunt dependent, and shunt-related thrombosis or other shunt-related problems would therefore be disastrous. This may be one reason that optimal timing of cavopulmonary connection may differ between the cohorts. Also, the right ventricular morphology in the Norwood group, as opposed to the left ventricular morphology in the tricuspid atresia cohort, may also alter the optimal window for cavopulmonary connection. 


\section{Appendix E1}

\section{Procedural Characteristics at Palliation}

Details regarding procedural characteristics at palliation with a systemic-pulmonary arterial shunt or PA band are shown in Table E2. Balloon septostomy was performed in 23 patients. Palliation was performed in 149 patients: systemic-pulmonary arterial shunt in $96(64 \%)$, initial BDCPA in $36(24 \%)$, and PA trunk banding in $17(11 \%)$. One infant enrolled in 2004 was alive without any operation.

\section{Systemic-Pulmonary Arterial Shunt Characteristics}

Patients undergoing systemic-pulmonary arterial shunt placement were younger (median age 7 days) than those undergoing PA trunk banding (median age 47 days, $P=.004$ ) but had equivalent body weight. The operative approach for systemic shunt placement was through median sternotomy in 69 patients and thoracotomy in 27 patients. Subclavian artery origin was universally used in those undergoing thoracotomy. The carotid artery was used in 2 patients, both of whom had aberrant right subclavian artery, and central shunts originating from the aorta were used in those with hypoplastic PAs or small arch vessels that precluded their use. Of the 96 patients who underwent systemic-pulmonary arterial shunt placement, concomitant interruption of competing sources of pulmonary blood flow included ligation of the main PA trunk in 11 and ligation of a patent ductus arteriosus in 33. Shunts originating from the innominate artery were the most common $(65 \%)$ and when indexed to body weight were smaller than shunts originating from other locations $(1.15 \pm 0.24 \mathrm{~mm} / \mathrm{kg}$ vs $1.31 \pm 0.56 \mathrm{~mm} / \mathrm{kg}, P=$ $.07)$. At discharge, unadjusted postoperative oxygen saturation was $83 \%$ in those with innominate artery shunts versus $85 \%$ for those with shunts originating from other locations among the 85 patients $(89 \%)$ for whom data were available. By multivariable analysis, after controlling for shunt diameter and other sources of pulmonary blood flow, this difference was $2.9 \pm 1.4(P=.04)$, indicating that non-innominate artery shunts resulted in higher total pul- monary blood flow. Eight patients required subsequent systemicpulmonary arterial shunt revision or replacement for shunt thrombosis $(n=4)$, failed BDCPA $(n=2)$, or other technical considerations $(\mathrm{n}=2)$.

\section{PA Banding Characteristics}

PA trunk banding $(n=17)$ occurred exclusively in patients with unrestricted pulmonary blood flow. Previous PA trunk banding was not associated with the development of a restrictive VSD or systemic outflow tract obstruction at the pre-BDCPA evaluation.

\section{Procedural Characteristics at Cavopulmonary Connection}

Details regarding procedural characteristics at BDCPA are listed in Table E3. The median age at BDCPA was 6 months (range 2.3 months-1.9 years). BDCPA followed systemic-pulmonary arterial shunt placement in 76 patients, followed PA trunk banding in 16, and was the initial procedure in 36 patients. BDCPA was used in $83 \%$, with a hemi-Fontan procedure performed in $17 \%$. Pulmonary arterioplasty (right PA greater than left PA) was the most frequent concomitant procedure and was slightly more prevalent among those with previous systemic shunt placement (17\% vs $9 \%, P$ not significant).

\section{Procedural Characteristics at Fontan Completion}

Details regarding the procedural characteristics at Fontan completion are listed in Table E4. Fontan completion occurred as a single-stage operation in 2 patients after systemic-pulmonary arterial shunt placement and in 74 patients after BDCPA. The median age at Fontan completion was 2.5 years (range 1.1-4.9 years), and the median interval from BDCPA was 1.9 years (range 6 months -3.6 years). The extracardiac Fontan procedure was used in $53 \%$ and the lateral tunnel was used in $35 \%$. Fenestration was used in $63 \%$. 


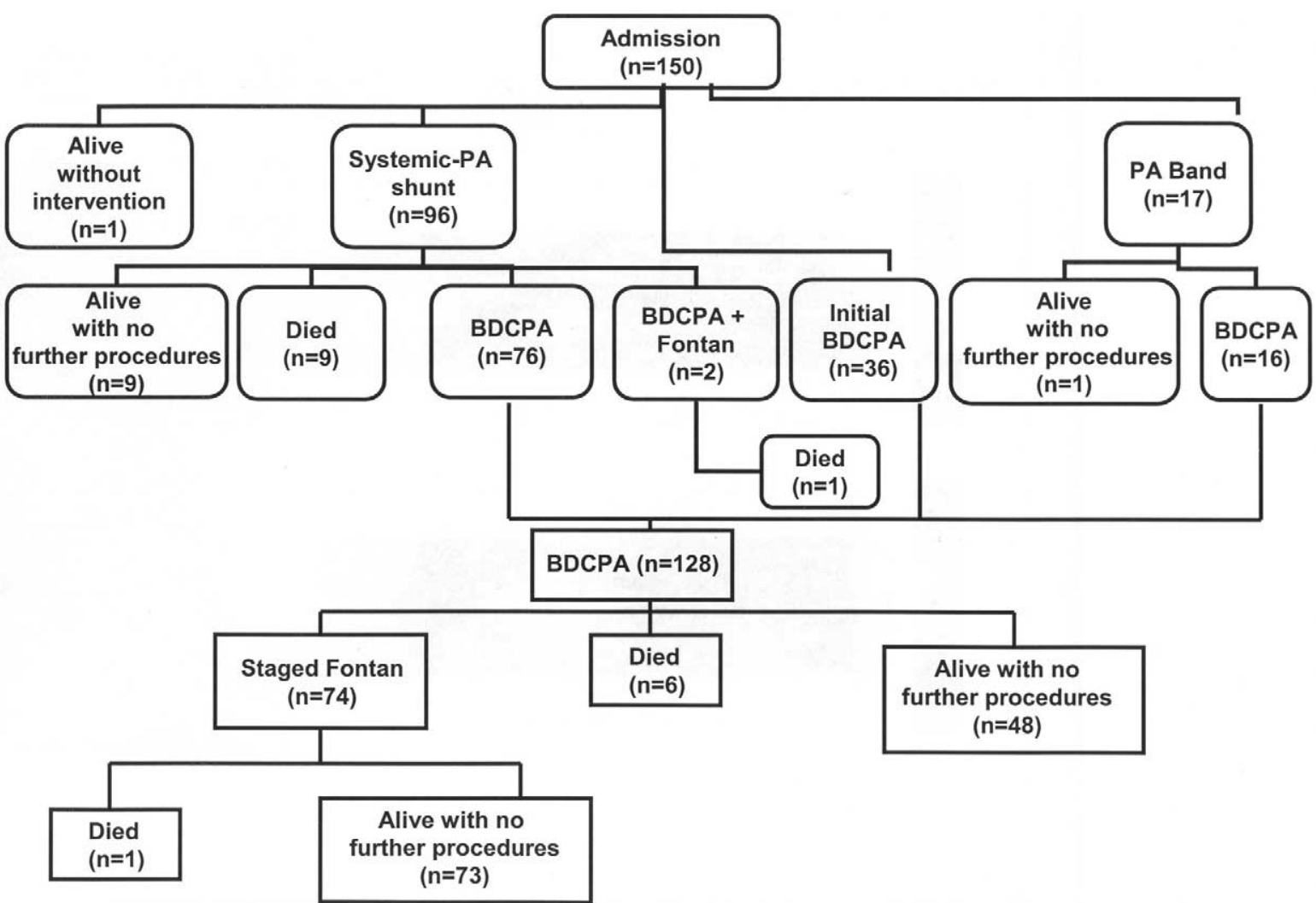

Figure E1. Flow chart depicting events from diagnosis among 150 patients with tricuspid atresia. One patient enrolled during 2004 remains alive without operation. All other patients underwent palliation consisting of systemic-pulmonary arterial shunt, PA banding, or initial BDCPA. Nine deaths occurred after systemic-PA shunt placement, and 128 patients had BDCPA. Of those who had BDCPA, 7 died: 6 before Fontan completion and 2 after Fontan conversion. Seventy-four patients underwent staged Fontan completion. Two patients had single-stage Fontan completion after systemic-pulmonary arterial shunt placement. 
TABLE E1. Initial patient characteristics $(n=150)$

\begin{tabular}{|c|c|c|c|}
\hline Variable & Value & Deaths (n = 17) & No. missing \\
\hline \multicolumn{4}{|l|}{ Demographic characteristics } \\
\hline Birth weight ( $\mathrm{kg}$, median and range) & $3.2(1.0-4.7)$ & & 28 \\
\hline Age at diagnosis ( $d$, median and range) & $0(0-62)$ & & 0 \\
\hline Admission age (y, median and range) & $0(0-1.7)$ & & 5 \\
\hline Sex (female/male) & $73: 77$ & $7: 10$ & 0 \\
\hline Noncardiac anomaly (No.) & $27(18 \%)$ & $6(11 \%)$ & 0 \\
\hline \multicolumn{4}{|l|}{ Morphologic characteristics } \\
\hline \multicolumn{4}{|l|}{ Type of tricuspid atresia (No.) } \\
\hline Type la & $28(19 \%)$ & $2(1 \%)$ & 1 \\
\hline Type Ib & $80(54 \%)$ & $10(7 \%)$ & 1 \\
\hline Type Ic & $41(28 \%)$ & $5(3 \%)$ & 1 \\
\hline Right aortic arch (No.) & $2(2 \%)$ & $0(0 \%)$ & 34 \\
\hline Patent ductus arteriosus (No.) & $93(71 \%)$ & $11(8 \%)$ & 19 \\
\hline Restrictive atrial septal defect (No.) & $11(9 \%)$ & $0(0 \%)$ & 27 \\
\hline Left superior vena cava (No.) & $14(13 \%)$ & $1(1 \%)$ & 38 \\
\hline Aortic valve stenosis (No.) & $4(3 \%)$ & $2(1 \%)$ & 15 \\
\hline Aortic valve regurgitation (No.) & $13(10 \%)$ & $3(2 \%)$ & 15 \\
\hline Mitral valve regurgitation (No.) & $32(24 \%)$ & $7(5 \%)$ & 16 \\
\hline Branch PA stenosis (No.) & $2(1 \%)$ & $0(0 \%)$ & 34 \\
\hline Aberrant right subclavian artery (No.) & $2(1 \%)$ & $1(1 \%)$ & 0 \\
\hline Anomalous pulmonary venous drainage (No.) & $1(1 \%)$ & $1(1 \%)$ & 27 \\
\hline VSD size $(m m$, mean $\pm S D)$ & $5.5 \pm 2.5$ & & 91 \\
\hline Left PA $Z$ score (median and range) & $! 1$ (!6 to 4) & & 56 \\
\hline Right PA $Z$ score (median and range) & $! 1$ (!6 to 4) & & 55 \\
\hline Combined mean $Z$ score ${ }^{*}$ (median and range) & $! 1$ (!5 to 3$)$ & & 56 \\
\hline Pulmonary valve annulus $(\mathrm{mm}$, mean $\pm \mathrm{SD})$ & $6.5 \pm 2.2$ & & 95 \\
\hline
\end{tabular}

*Combined mean $Z$ score $=$ (right PA $Z$ score + left PA $Z$ score $) / 2$. 
TABLE E2. Procedural characteristics at palliation $(n=149)$

\begin{tabular}{|c|c|c|}
\hline Variable & Value & No. missing \\
\hline \multicolumn{3}{|l|}{ Type of palliation (No.) } \\
\hline Systemic-pulmonary arterial shunt & $96(64 \%)$ & 0 \\
\hline PA band & $17(11 \%)$ & 0 \\
\hline Cavopulmonary connection & $36(24 \%)$ & 0 \\
\hline \multicolumn{3}{|c|}{ Systemic-pulmonary arterial shunt characteristics ( $\mathrm{n}=96$ ) } \\
\hline Age ( $d$, median and range) & $7(0-138)$ & 0 \\
\hline Weight $(\mathrm{kg}$, mean $\pm \mathrm{SD})$ & $3.4 \pm 1.0$ & 9 \\
\hline \multicolumn{3}{|l|}{ Location of shunt origin (No.) } \\
\hline Innominate artery & $62(65 \%)$ & 0 \\
\hline Subclavian artery & $28(29 \%)$ & 0 \\
\hline Other site & $6(6 \%)$ & 0 \\
\hline Operative approach (No.) & & 0 \\
\hline Median sternotomy & $69(72 \%)$ & 0 \\
\hline Thoracotomy & $27(28 \%)$ & 0 \\
\hline Indexed shunt size $(\mathrm{mm} / \mathrm{kg}$, mean $\pm \mathrm{SD})$ & $1.2 \pm 0.3$ & 15 \\
\hline Main PA ligation (No.) & $11(11 \%)$ & 0 \\
\hline Patent ductus arteriosus ligation (No.) & $33(35 \%)$ & 3 \\
\hline Preoperative saturation (\%, mean $\pm \mathrm{SD})$ & $74 \pm 18$ & 13 \\
\hline Postoperative saturation $(\%$, mean $\pm \mathrm{SD})$ & $85 \pm 11$ & 11 \\
\hline Cardiopulmonary bypass used (No.) & $16(17 \%)$ & 0 \\
\hline Circulatory arrest (No.) & $4(4 \%)$ & 0 \\
\hline \multicolumn{3}{|l|}{ PA band characteristics $(\mathrm{n}=17$ ) } \\
\hline Age ( $d$, median and range) & $47(0-14)$ & 0 \\
\hline Weight $(\mathrm{kg}$, mean $\pm \mathrm{SD})$ & $3.8 \pm 0.9$ & 3 \\
\hline
\end{tabular}

TABLE E3. Procedural characteristics at BDCPA $(n=128)$

\begin{tabular}{lcr}
\hline Variable & Value & No. missing \\
\hline Initial BDCPA (No.) & $36(28 \%)$ & 0 \\
Age (median and range) & $5.7 \mathrm{~m}(1.7 \mathrm{~m}-1.9 \mathrm{y})$ & 0 \\
Weight (kg, mean \pm SD) & $6.5 \pm 1.3$ & 12 \\
Type of BDCPA (No.) & & \\
$\quad$ Bidirectional Glenn shunt & $106(83 \%)$ & 0 \\
Hemi-Fontan procedure & $22(17 \%)$ & 0 \\
CPB time (min, mean \pm SD) & $68 \pm 40$ & 23 \\
Circulatory arrest used (No.) & $21(17 \%)$ & 3 \\
Postoperative saturation (\%, mean \pm SD) & $84 \% \pm 6 \%$ & 21 \\
Myocardial protection method (No.) & & 16 \\
Cardioplegia used & $45(40 \%)$ & 16 \\
No aortic crossclamp & $58(52 \%)$ & 16 \\
Fibrillation, no cardioplegia used & $9(8 \%)$ & 3 \\
Concomitant procedures (No.) & & 8 \\
Right pulmonary arterioplasty & $32(26 \%)$ & 0 \\
Left pulmonary arterioplasty & $19(15 \%)$ & 0 \\
Main PA band & $8(6 \%)$ & \\
Main PA ligation & $59(48 \%)$ & \\
\hline
\end{tabular}


TABLE E4. Characteristics at Fontan completion $(n=76)$

\begin{tabular}{lcc}
\hline Variable & Value & No. missing \\
\hline Single-stage Fontan (No.) & $2(3 \%)$ & 0 \\
Age (y, median and range) & $2.4(0.9-4.9)$ & 0 \\
Weight (kg, mean \pm SD) & $12.9 \pm 3.1$ & 9 \\
Type of Fontan procedure (No.) & & 1 \\
$\quad$ Extracardiac conduit & $40(53 \%)$ & 1 \\
$\quad$ Lateral tunnel & $35(47 \%)$ & 3 \\
Conduit material (No.) & & 3 \\
$\quad$ Polytetrafluoroethylene & $66(90 \%)$ & 3 \\
$\quad$ Allograft & $3(4 \%)$ & 1 \\
$\quad$ Pericardium & $4(5 \%)$ & 33 \\
Fenestration performed (No.) & $47(63 \%)$ & 38 \\
Fenestration size (mm, median and range) & $4(2-7)$ & 25 \\
Postoperative saturation (\%, mean \pm SD) & $93 \% \pm 6 \%$ & 0 \\
Cardiopulmonary bypass time (min, mean \pm SD) & $81 \pm 31$ & 74 \\
Circulatory arrest used (No.) & $7(9 \%)$ & $14(18 \%)$ \\
Main PA ligation (No.) & & \\
\hline
\end{tabular}

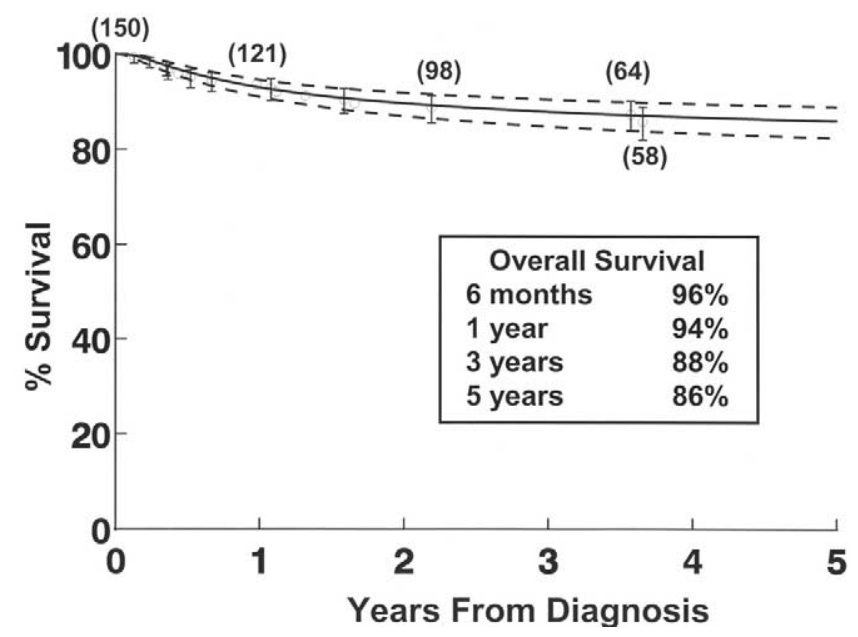

Figure E2. Overall time-related survival among 150 patients with tricuspid atresia. Solid lines represent parametric point estimates enclosed by $70 \%$ confidence intervals; circles with error bars represent nonparametric estimates; numbers in parentheses represent number of patients traced at that point. 


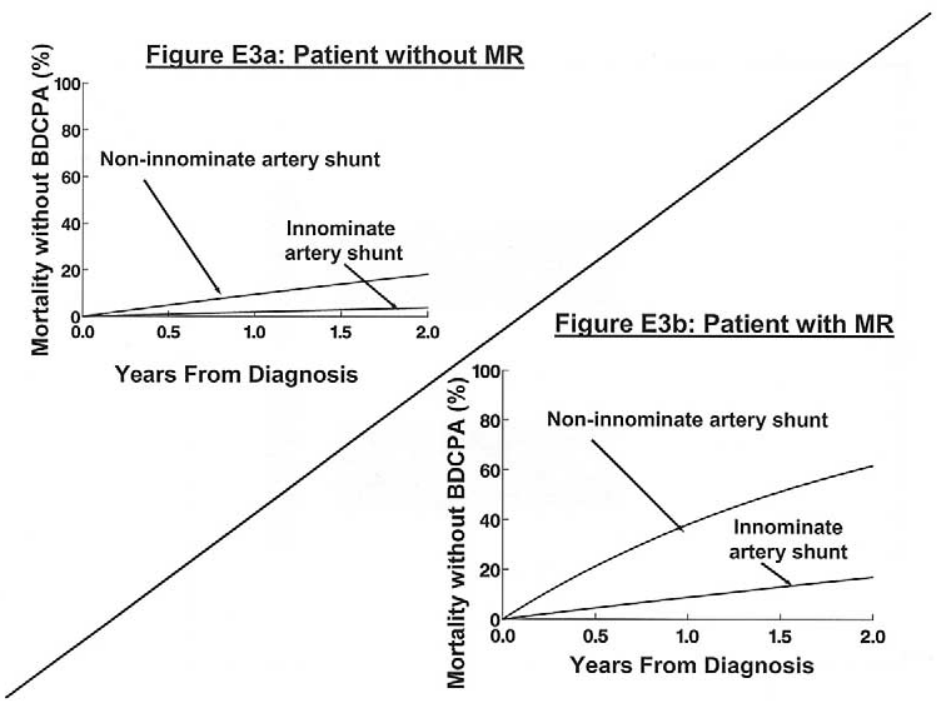

Figure E3. A, Predicted mortality without achievement of BDCPA stratified by systemic-pulmonary arterial shunt origin in absence of mitral valve regurgitation (MR). B, Predicted mortality without achievement of BDCPA stratified by systemic-pulmonary arterial shunt origin in presence of mitral valve regurgitation. Although noninnominate artery shunt origin results in decreased survival in both conditions, its unfavorable influence is greater in presence of significant mitral regurgitation. Solid lines represent continuous parametric estimates.

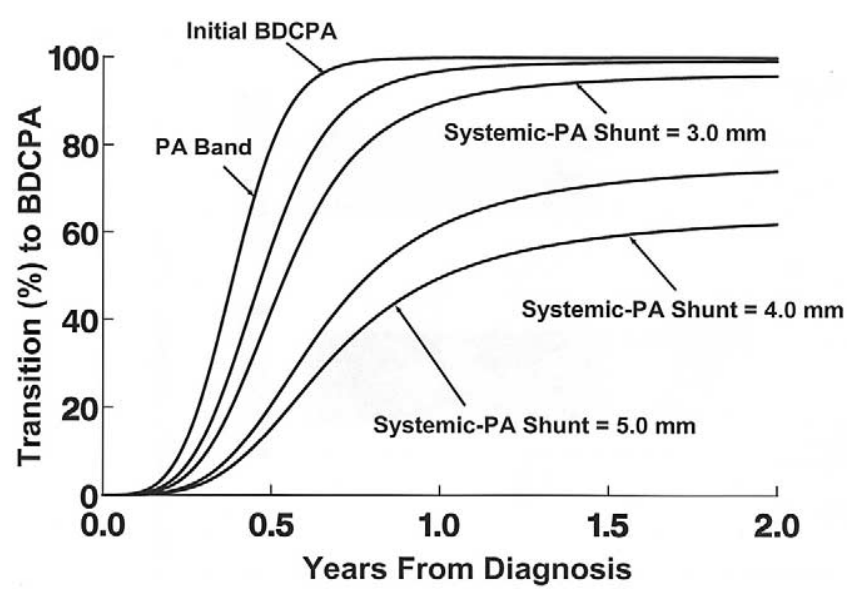

Figure E4. Predicted achievement of and transition rates to BDCPA from diagnosis stratified by type of palliation and size of initial systemic-pulmonary arterial shunt. Five lines represent five specific solutions to multivariable equation for achievement of BDCPA for hypothetic patient without additional anomalies admitted at 1 year of age. Mean values were used for other continuous predictors. For patients requiring systemic-pulmonary arterial shunt, placement of smaller diameter shunt is associated with greatest prevalence of patients reaching defined end state. Solid lines represent continuous parametric point estimates. 


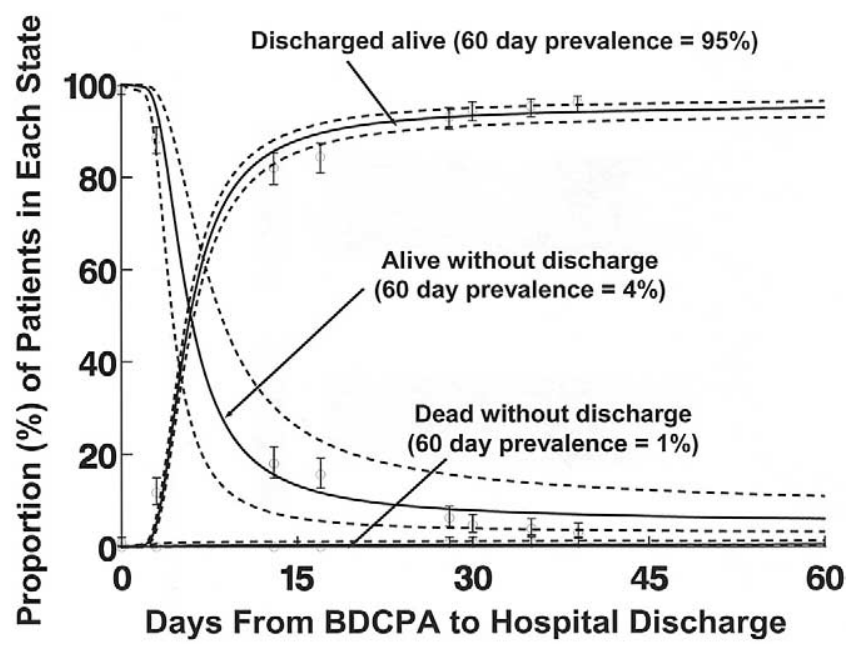

Figure E5. Competing risks diagram of events after BDCPA to hospital discharge. Median LOS was 6 days (range 3-351 days). Competing risks analysis showed that 60 days after BDCPA, 95\% were discharged alive, $4 \%$ remained alive in hospital, and $1 \%$ died without discharge. 